\title{
Can mutated stem cells produce tumours?
}

Which cells need to be mutated in order for solid tumours to fully develop? Many have postulated that mutation of a normal tissue stem cell might be required for both tumour initiation and progression, whereas others have argued that mutations in progenitor cells can induce stem cell-like qualities, giving rise to longlived and clinically manifest disease. Two papers recently published in Nature add thought-provoking data to this debate.

Hans Clevers and colleagues have recently identified cells within the crypts of the small intestine and colon expressing LGR5 to be long-lived stem cells. Having established that tumours form only when the tumour suppressor $A p c$ is deleted in a rare population of cells in the crypt, the authors crossed transgenic mice expressing floxed $A p c$ alleles with knock-in mice expressing enhanced green fluorescent protein (EGFP) and tamoxifen-regulated Cre recombinase under the control of the Lgr5 promoter. Activation of the Cre recombinase resulted in EGFPpositive cells expressing increased levels of $\beta$-catenin (which is regulated by APC) in a few cells in the base of the crypt. These cells continued to
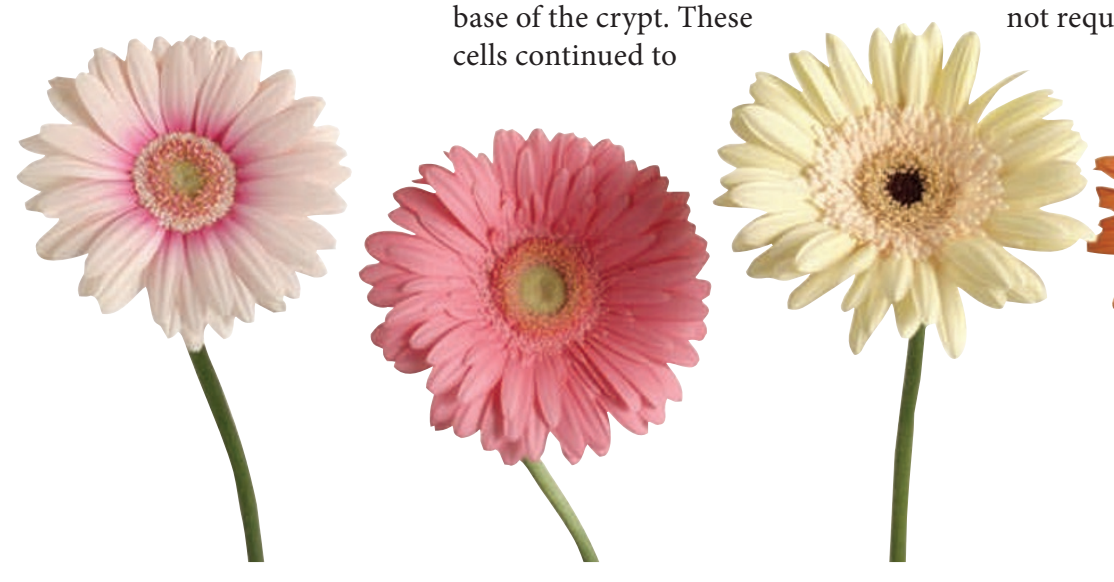

Unlike other tissues in the Prom $1^{\mathrm{C}-\mathrm{L}}$-heterozygous mice, PROM1 expression was restricted to the stem cell compartment in the small intestine. The distribution of Prom 1 and Lgr5 overlapped: $75 \%$ of Lgr5 mRNA-positive cells in Prom $^{+/ C-L}$ mice also expressed nuclear LacZ. Crossing Prom $1^{+/ C-L}$ mice with Cre-dependent Rosa26 yellow fluorescent protein (YFP)-expressing mice, thereby constitutively activating YFP in PROM1-expressing cells and their progeny after injection of tamoxifen, showed that PROM1 cells can give rise to all cells of the small intestinal epithelium. Crossing these mice with mice expressing an inducible oncogenic form of $\beta$-catenin resulted in tumour formation in these animals. Interestingly, only $7 \%$ of the cells within these tumours remained PROM1-positive; the authors suggest that these might be a subpopulation of transformed stem cells.

Both papers indicate that a single mutation in normal intestinal stem cells can give rise to tumours, as has been suggested. It is interesting that, although LGR5 and PROM1 seem to mark similar stem cells in the small intestine, PROM1 does not mark colonic stem cells, whereas LGR5 does. This illustrates the need to clearly define markers and their limitations if we are to begin to understand the contribution of normal tissue stem cells and cancer stem cells to tumorigenesis.

Nicola McCarthy

ORIGINAL RESEARCH PAPER Barker, N. et al. Crypt stem cells as the cells of origin of intestinal cancer. Nature 17 Dec 2008 (doi 10.1038/ nature07602)|Zhu, L. et al. Prominin 1 marks intestinal stem cells that are susceptible to neoplastic transformation. Nature 17 Dec 2008 (doi 10.1038/nature07589) 